\title{
New Tools in Bio-NMR: Indirect Detection of Nitrogen-14 in Solids
}

\author{
Simone Ulzega $\S^{\star}$ \\ §SCS Mettler-Toledo Award Winner (Oral Presentation)
}

\begin{abstract}
This paper presents an overview of recently developed methods for the indirect detection of ${ }^{14} \mathrm{~N}$ nuclei (spin $I=1$ ) in spinning solids by nuclear magnetic resonance spectroscopy. These methods exploit the transfer of coherence from a neighboring 'spy' nucleus with spin $S=1 / 2$, such as ${ }^{13} \mathrm{C}$ or ${ }^{1} \mathrm{H}$, to single- or double-quantum transitions of ${ }^{14} \mathrm{~N}$ nuclei. The two-dimensional correlation methods presented here are closely related to the wellknown heteronuclear single- and multiple-quantum correlation (HSQC and HMQC, respectively) experiments, already widely used for the investigation of molecules in liquids. Nitrogen-14 NMR spectra exhibit powder patterns characterized by second- and third-order quadrupolar couplings which can provide important information about structure and dynamics of molecules in powder samples.
\end{abstract}

Keywords: Dynamics in solids · Magic angle spinning (MAS) · Nitrogen-14 NMR spectroscopy ·

Quadrupolar nuclei

\section{Introduction}

Nuclear magnetic resonance (NMR) spectroscopy is a powerful and versatile tool for the investigation of matter through the measurement of nuclear spin interactions which has found applications in numerous disciplines of science. In particular, solidstate NMR has become a very active area of research in structural investigations of samples that are not accessible to liquidstate NMR or that do not crystallize, with important applications in both biochemistry and material science. ${ }^{[1-3]}$ Recently, high-resolution solid-state NMR has been improved significantly by the advent of fast magic-angle spinning (MAS) probes (up to $70 \mathrm{kHz}$ ) and new heteronuclear decoupling schemes using low-power radiofrequency $(r f)$ irradiation. ${ }^{4-6]}$

So far, nitrogen-14 NMR has failed to become a routine spectroscopic technique, like ${ }^{1} \mathrm{H},{ }^{13} \mathrm{C}$ and ${ }^{15} \mathrm{~N}$ NMR, despite the fundamental structural and functional role of nitrogen in proteins and nucleic acids and its ubiquitous presence in many materials. Nevertheless, the favorable isotopic abundance (99.6\%) and gyromagnetic ratio $\left(\sim 70 \%\right.$ of $\left.{ }^{15} \mathrm{~N}\right)$ make ${ }^{14} \mathrm{~N}$ a potentially

${ }^{*}$ Correspondence: Dr. S. Ulzega Institut des Sciences et Ingénierie Chimiques Ecole Polytechnique Fédérale de Lausanne $\mathrm{CH}-1015$ Lausanne

Tel.: +41216939386

Fax: + 41216939435

E-mail: simone.ulzega@epfl.ch attractive spectroscopic probe. In contrast to nuclei with spin $S=1 / 2\left({ }^{1} \mathrm{H},{ }^{13} \mathrm{C},{ }^{15} \mathrm{~N}\right),{ }^{14} \mathrm{~N}$ has $\operatorname{spin} I=1$ and therefore a nuclear quadrupole moment $Q$. The interaction between the latter and the electric field gradient $V$ at the site of the nucleus can be characterized by an asymmetry parameter $\eta_{q}=\left(V_{v y}-V_{x x}\right) /$ $V_{z 7}\left(0<\eta_{q}<1\right)$ and by a quadrupole coupling constant $C_{O}=e Q V_{z z} / h$, which can be as large as a few MHz. In the absence of magnetic fields, ${ }^{14} \mathrm{~N}$ quadrupole interactions can be characterized by nuclear quadrupole resonance $(\mathrm{NQR}),{ }^{[7,8]}$ whereas in a static magnetic field the strong quadrupole interaction leads to NMR spectra that often span a width of several MHz. Such broad spectra are characterized by a very low resolution, they are difficult to excite uniformly, and require broad receiver bandwidths, which significantly limit the sensitivity.

In solid-state NMR it is common practice to spin a powder sample at the magic angle $\theta_{m}=54.7^{\circ}$ (magic angle spinning, MAS) to average out the first-order components of second-rank tensor interactions, such as the dipolar couplings, the anisotropic part of the chemical shift, and the quadrupole interaction. ${ }^{[9]}$ Nevertheless, under MAS ${ }^{14} \mathrm{~N}$ spectra can feature hundreds of spinning sidebands that may span several megahertz. ${ }^{[10]}$ The envelope of these sidebands depends critically on several parameters, such as the accurate adjustment of $\theta_{r}$, the amplitude and length of the ${ }^{14} \mathrm{~N} r f$ pulses and the bandwidths of probe and receiver systems. ${ }^{[11]}$ Because of the large quadrupole couplings $\left(C_{\rho} \sim 1-3 \mathrm{MHz}\right)$, the (direct) detection of ${ }^{14} \mathrm{~N}$ NMR spectra is extremely challenging.
In this article it is shown that ${ }^{14} \mathrm{~N}$ NMR of solid biological samples is feasible thanks to recently developed methods for two-dimensional correlation experiments that combine MAS with indirect detection of ${ }^{14} \mathrm{~N}$ via a neighboring spy nucleus with favorable spin $S=1 / 2\left({ }^{1} \mathrm{H}\right.$ or $\left.{ }^{13} \mathrm{C}\right) \cdot{ }^{[9,11-14]}$ These new methods are closely related to early methods for the indirect detection of ${ }^{15} \mathrm{~N}$ in liquid-state NMR ${ }^{[15,16]}$ known as heteronuclear single- and multiple-quantum correlation (HSQC and HMQC, respectively).

Besides structural information, NMR can provide important information about inter- and intramolecular dynamic processes that can affect spectral features such as lineshapes and relaxation times when their intrinsic rates are matched with characteristic frequencies of the experiment. Dynamic processes can be probed over a wide range of timescales, ${ }^{[17]}$ ranging approximately from picoseconds to seconds. Solid-state NMR has recently turned out to be an attractive tool to study dynamics. ${ }^{[18-23]}$ Solid-state methods exploit spectral changes associated with the modulation of anisotropic interactions, e.g. quadrupolar interactions, induced by motions. This article shows that ${ }^{14} \mathrm{~N}$ solidstate NMR spectroscopy can yield information about local dynamics in nitrogencontaining solids. ${ }^{[24]}$

\section{Methods}

The methods for the indirect detection of ${ }^{14} \mathrm{~N}$ exploit the transfer of coherence back and forth between single- (SQ) 
and double-quantum (DQ) coherences of ${ }^{14} \mathrm{~N}$ (spin $I=1$ ) and SQ coherences of a spy nucleus $(S=1 / 2)$, which can be either ${ }^{13} \mathrm{C}$ or ${ }^{1} \mathrm{H}$. The methods using ${ }^{13} \mathrm{C}$ detection were introduced independently by $\mathrm{Gan}^{[13]}$ at the National High Magnetic Field Laboratory, Florida, and Cavadini et al. ${ }^{[12]}$ at the EPFL, while ${ }^{1} \mathrm{H}$ detection was recently demonstrated by Cavadini et al. ${ }^{[14]}$ at the EPFL. The latter approach has the advantage of enhanced sensitivity and does not require ${ }^{13} \mathrm{C}$ isotopic enrichment, although the linewidths can be significantly broadened by the strong homonuclear proton-proton couplings. Henceforth we shall focus on proton-detected experiments. The method discussed in this paper was built on the scheme known in liquid-state NMR as HMQC. The remarkably simple pulse sequence is shown in Fig. 1a. The coherence transfer pathway between spins $I$ and $S$ is shown schematically in Fig. 1b. A variant of the scheme, i.e. the HSQC experiment, will be briefly discussed at the end of this section.

In the pulse sequence of Fig. 1a, the first $\pi / 2$ pulse (time point a) is applied to excite transverse proton magnetization, represented in terms of Cartesian product operators by $S_{x}$ or equivalently in terms of tensor spin operators by $\left(\mathrm{T}_{1,-1}^{S}-\mathrm{T}_{1,1}^{S}\right) / 2$. The following excitation delay, $\tau_{\text {exc }}$, allows one to transfer coherence from spin $S$ to spin $I$ and thus create SQ or DQ coherences involving both nuclei $S$ and $I$. Several mechanisms can be exploited. ${ }^{[9]}$ The large quadrupole coupling of nitrogen nuclei prevents a proper quantization of spin $I$ along the $z$ direction of the main static field $\left(\mathrm{B}_{0}\right)$. Consequently, the first-order heteronuclear dipolar interaction between spins $S$ and $I, \mathrm{D}\left({ }^{14} \mathrm{~N}, S\right)$, cannot be averaged out completely by MAS. This effect leaves a residual dipolar splitting (RDS) that depends on the mutual orientation of the nuclear quadrupole and dipolar tensors and on the orientation of each crystallite of the powder sample with respect to the rotor axis. The coupling $\mathrm{D}_{\mathrm{RDS}}\left({ }^{14} \mathrm{~N}, S\right)$ is inversely proportional to the static field $B_{0}$. The RDS is in fact due to a second-order quadrupole-dipole cross effect. The RDS splits the resonance of spin $S$ into a doublet, the components of which feature typical quadrupolar powder patterns. ${ }^{[9]}$ The most intense component of this doublet can be further split into a doublet by the heteronuclear scalar coupling $\mathrm{J}\left({ }^{14} \mathrm{~N}, S\right)$, which is usually negligible for ${ }^{13} \mathrm{C}$ nuclei but may be significant for protons. For instance, one has $\mathrm{J}\left({ }^{14} \mathrm{~N},{ }^{13} \mathrm{C}\right)$ in the range $3-8 \mathrm{~Hz}$ in L-alanine, whereas in a rigid $\mathrm{CO}^{14} \mathrm{~N}^{1} \mathrm{H}^{\mathrm{N}} \mathrm{C}$ group such as in $\mathrm{N}$-acetylglycine $\mathrm{J}\left({ }^{14} \mathrm{~N},{ }^{1} \mathrm{H}\right)$ can be as large as $\sim 65 \mathrm{~Hz}$. In N-acetylglycine the coupling $\mathrm{D}_{\mathrm{RDS}}\left({ }^{14} \mathrm{~N},{ }^{1} \mathrm{H}\right)$ ranges approximately from 600 to $300 \mathrm{~Hz}$ when $\mathrm{B}_{0}$ is varied from 9.4 to $18.8 \mathrm{~T}$. Besides RDS

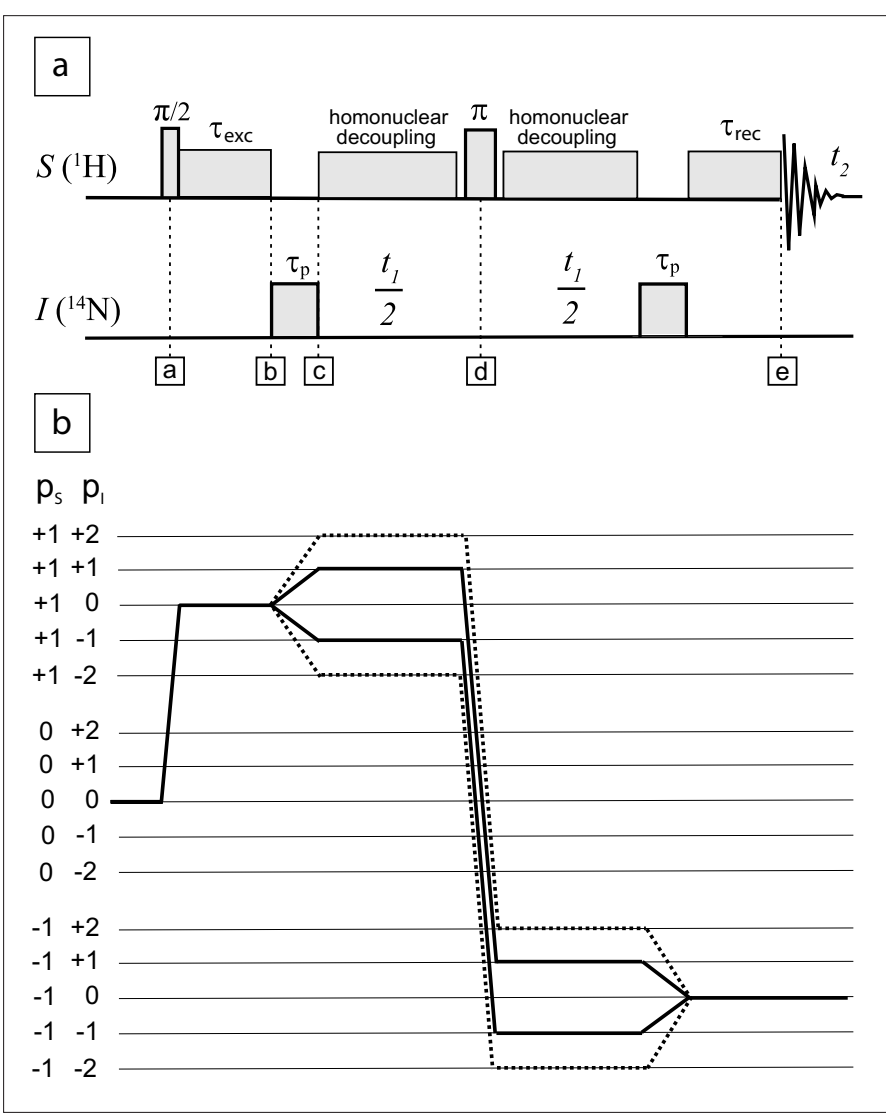

Fig. 1. a) Pulse sequence for the indirect observation of ${ }^{14} \mathrm{~N} \mathrm{SQ}$ and DQ spectra in solids rotating at the magic angle (MAS solid-state NMR). The pulse sequence is based on the HMQC scheme, as explained in the text, and exploits detection via protons. b) Coherence transfer pathway between spins $S\left({ }^{1} \mathrm{H}\right)$ and $/$ $\left({ }^{14} \mathrm{~N}\right)$ for $\mathrm{SQ}$ (solid line) and DQ (dotted line) coherences.

and J-couplings, the transfer of coherence during $\tau_{\text {exc }}$ can be achieved via recoupled heteronuclear dipolar interactions. Recoupling can be achieved using rotary resonance, ${ }^{[25-28]}$ i.e. by applying a continuous $r f$ field to spin $S$ with an amplitude that is a multiple of the rotor spinning frequency, $\omega^{S}=\mathrm{n} \omega_{\text {rot }}$. Rotary resonance with $\mathrm{n}=2$ (e.g. $\omega_{\text {rot }} /(2 \pi)=30 \mathrm{kHz}$ and $\omega_{1}^{S} /(2 \pi)=60$ $\mathrm{kHz}$ ) has been successfully used for the indirect proton-detection of ${ }^{14} \mathrm{~N}$ in glycine in natural abundance. ${ }^{[28]}$ Alternatively, one can use rotor-synchronized symmetrybased RN sequences ${ }^{[29,30]}$ to recouple heteronuclear dipolar $I-S$ interactions while simultaneously decoupling homonuclear ${ }^{1} \mathrm{H}-{ }^{1} \mathrm{H}$ interactions. ${ }^{[31]}$

At the end of the delay time $\tau_{\text {exc }}$ the mechanisms described above lead to a state (time point $b$ in Fig. 1a) that can be described by a superposition of doubly- and singly antiphase operators $S_{\mathrm{x}} I_{\mathrm{z}}^{2}$ and $S_{\mathrm{x}} I_{\mathrm{z}}$. In terms of spin tensor operators one may consider a superposition of $\mathrm{T}_{1, \mathrm{~m}}^{S} \mathrm{~T}_{2,0}^{I}$ and $\mathrm{T}_{1, \mathrm{~m}}^{S} \mathrm{~T}_{1,0}^{I}$, with $\mathrm{m}= \pm 1$. This state is characterized by a coherence order $\mathrm{p}_{S}=+1$ for spin $S$ and $\mathrm{p}_{I}=0$ for spin $I$. The next step of the sequence allows one to create coherences of spin $I$ of order \pm 1 (see Fig. 1b). An $r f$ pulse applied in the middle of the ${ }^{14} \mathrm{~N}$ spectrum leads to the partial conversion of $\mathrm{T}_{1, \mathrm{~m}}^{S} \mathrm{~T}_{1,0}^{I}$ into $\mathrm{T}_{1, \mathrm{~m}}^{S} \mathrm{~T}_{1, \pm 1}^{I}$ and of $\mathrm{T}^{S}{ }_{1, \mathrm{~m}} \mathrm{~T}_{2,0}^{I}$ into $\mathrm{T}^{S_{1, \mathrm{~m}}^{1, \mathrm{~m}}} \mathrm{~T}_{2,+1}^{1,0}$, both involving ${ }^{14} \mathrm{~N} \mathrm{SQ}^{1, \mathrm{~m}}$ coherences of rank 1 and 2, respectively, and of $\mathrm{T}_{1, \mathrm{~m}}^{S} \mathrm{~T}_{2,0}^{I}$ into $\mathrm{T}_{1, \mathrm{~m}}^{S} \mathrm{~T}_{2, \pm 2}^{I}$, which involves ${ }^{14} \mathrm{~N}$ DQ coherences (time point $\mathrm{c}$ in Fig. 1). It is worth remarking that all of the above terms actually describe heteronuclear multiplequantum (MQ) coherences if $m \neq 0$. For the sake of clarity, we shall speak here of ${ }^{14} \mathrm{~N}$ SQ or DQ coherences when the terms $\mathrm{T}_{\mathrm{k}+1}^{I}$ $(\mathrm{k}=1,2)$ or $\mathrm{T}_{2, \pm 2}^{I}$ are involved, regardless of the participation of spin $S$ (represented by terms like $\mathrm{T}^{S}$ ) to form heteronuclear coherences. The length $\tau_{\mathrm{p}}$ of the ${ }^{14} \mathrm{~N}$ pulse must be optimized empirically. It was shown ${ }^{[11]}$ that a compromise $\tau_{\mathrm{p}} \sim(1 / 2) \tau_{\text {rot }}$, where $\tau_{\text {rot }}$ is the rotor period, allows one to excite efficiently both SQ and DQ coherences in the same experiment, relying on proper phase cycles to separate them later on. For instance, in ref. [11] it is shown with a sample of L-alanine at a MAS rate of $30 \mathrm{kHz}$ and using an $r f$ amplitude $\mathrm{v}_{1}\left({ }^{14} \mathrm{~N}\right)=$ $57 \mathrm{kHz}$ that $\tau_{\mathrm{p}}$ (SQ) $\sim 11 \mu \mathrm{s}$ and $\tau_{\mathrm{p}}$ (DQ) $22 \mu \mathrm{s}$, leading to an optimum compromise $\tau_{\mathrm{p}} \sim 16 \mu \mathrm{s} \sim(1 / 2) \tau_{\text {rot }}$.

The coherences $\mathrm{T}^{S}{ }_{1, \mathrm{~m}} \mathrm{~T}_{\mathrm{k}, \mathrm{q}}^{I}$ described above are then allowed to evolve freely during the evolution interval $t_{1}$, at the end of which all coherences are symmetrically reconverted into observable SQ coherences $\mathrm{T}_{1, \mathrm{~m}}^{S}$ (time point e in Fig. 1), and the free induction decay (FID) of the latter in the static field $\mathrm{B}_{0}$ is then detected (time interval $t_{2}$ ). The reconversion interval $\tau_{\text {rec }}$ has typically the same duration as the excitation interval $\tau_{\text {exc }}$. A $\pi$-pulse is applied to the $S$ spy nuclei in the middle of the evolution interval $t_{1}$ (time point $d$ ) to generate a spin-echo and thus refocus the offsets of $S$ nuclei and attenuate the decay due to field 


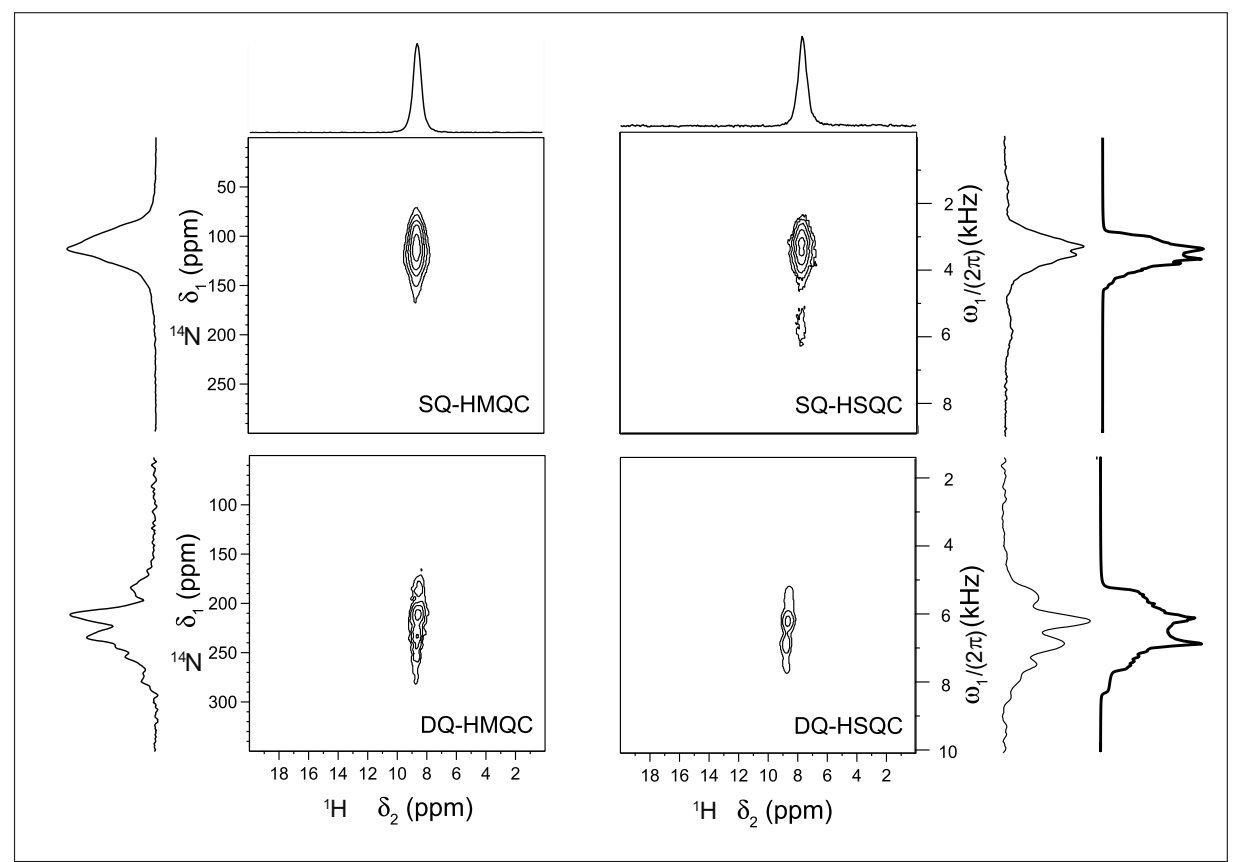

inhomogeneities of the observable $\mathrm{T}^{S}{ }_{1, \mathrm{~m}}$. In addition, we have recently shown that the inclusion of decoupling sequences, such as rotor-synchronized symmetry-based RN sequences, during the evolution time $t_{1}$ (as shown in Fig. 1a) may lead to an enhancement of the resolution of both SQ and DQ ${ }^{14} \mathrm{~N}$ spectra by reducing the broadening effect of the strong homonuclear ${ }^{1} \mathrm{H}-{ }^{1} \mathrm{H}$ dipolar couplings. ${ }^{32]}$ The scheme shown in Fig. 1 is repeated $N$ times with different evolution times $\mathrm{t}_{1}$ as in standard 2Dcorrelation experiments, i.e. $\mathrm{t}_{1}=\mathrm{n} \Delta \mathrm{t}_{1}(\mathrm{n}=$ $1,2, \ldots, N)$, with rotor-synchronized increments $\Delta \mathrm{t}_{1}=\tau_{\text {rot }}{ }^{[9]}$ It is important to stress the importance of a precise synchronization of the increments $\Delta \mathrm{t}_{1}$ with the MAS period. It is well-known that nitrogen-14 spectra under MAS are characterized by large families of spinning sidebands. The envelope of these sidebands is determined by the dominant first-order quadrupole interaction and can thus extend over several megahertz. The synchronization of $\Delta \mathrm{t}_{1}$ and $\tau_{\text {rot }}$ causes the sidebands to coincide in the $\omega_{1}$ (nitrogen) dimension with the centerbands, which depend on the second-order quadrupole interaction and typically span a range on the order of a few kilohertz. The synchronization also allows one to obtain pure-phase spectra in the indirect (nitrogen) dimension $\omega_{1}$. Indeed, Fig. $1 \mathrm{~b}$ shows that both SQ and DQ coherences are characterized by two mirror-image coherence transfer pathways with coherence orders $\mathrm{p}_{I}= \pm 1$ and $\mathrm{p}_{I}= \pm 2$, respectively. This allows one to obtain pure two-dimensional absorption lineshapes. It was shown ${ }^{[11]}$ that the two-way coherence transfer $(S \rightarrow I \rightarrow S)$ efficiencies of the scheme discussed above for SQ and DQ coherences of L-alanine were $\sim 16 \%$ and $\sim 12 \%$, respectively.
An important alternative to the HMQClike experiment described above is the HSQC scheme, ${ }^{[9,33]}$ characterized by two additional $\pi / 2$ pulses applied to spin $S$ at the beginning and at the end of the evolution period. These pulses convert the coherence $\mathrm{T}^{S}{ }_{1, \mathrm{~m}} \mathrm{~T}_{\mathrm{k}, \mathrm{q}}^{I}(\mathrm{~m}= \pm 1)$ into $\mathrm{T}^{S}{ }_{1,0} \mathrm{~T}_{\mathrm{k}, \mathrm{q}}^{I}$ and viceversa. This variant of the experiment has the advantage of being insensitive to the fast transverse relaxation of the coherences $\mathrm{T}^{S}{ }_{1, \mathrm{n}}$ $(\mathrm{m}= \pm 1)$ during the evolution interval, so that the lineshapes in the indirect $\omega_{1}$ dimension turn out to be determined only by the (negligible) longitudinal relaxation of the (longitudinal) term $\mathrm{T}_{1,0}^{S}$. Proton-detected HSQC experiments typically show a significant gain in resolution. However, the phase cycling needed for HSQC leads to a two-fold loss of the $\mathrm{S} / \mathrm{N}$ ratio compared to HMQC for the same number of scans.

Examples of ${ }^{14} \mathrm{~N}$ NMR spectra obtained by indirect detection via protons using both HMQC and HSQC schemes are shown in Fig. 2.

\section{Applications: A Study of Local Dynamics}

Solid-state NMR is a remarkable tool to probe dynamic processes on micro- and millisecond timescales. Recently, dynamics have been characterized by comparing ${ }^{2} \mathrm{H}(I=1)$ SQ and DQ spectra obtained under MAS. ${ }^{[19,22]}$ It is known that the combination of MAS and rotor-synchronization averages out first-order anisotropic interactions such as dipolar couplings, chemical shift anisotropy and quadrupolar interactions. However, when dynamic processes occur on timescales matching with one of these anisotropic interactions, an
Fig. 2. Proton-detected experimental HMQC and HSQC spectra showing ${ }^{14} \mathrm{~N} \mathrm{SQ}$ and $\mathrm{DQ}$ signals of the ${ }^{14} \mathrm{~N}^{1} \mathrm{H}_{3}{ }^{+}$ammonium group in partially deuterated glycine $\left[\mathrm{D}_{2}\right] \mathrm{NH}_{3}{ }^{+} \mathrm{CD}_{2} \mathrm{COO}$. A sample of $11 \mu \mathrm{l}$ was spun in a $2.5 \mathrm{~mm}$ rotor at $30.03 \mathrm{kHz}$ in a static field of $9.4 \mathrm{~T}(28.9$ and $400 \mathrm{MHz}$ for ${ }^{14} \mathrm{~N}$ and ${ }^{1} \mathrm{H}$, respectively). For the $S Q$ spectra, the ${ }^{14} \mathrm{~N}$ pulse lengths were $\tau_{\mathrm{p}}=12 \mu \mathrm{s}$ and $\tau_{\text {exc }}=\tau_{\text {rec }}=1 \mathrm{~ms}$. For the DQ spectra, $\tau_{\mathrm{p}}=35 \mu \mathrm{s}$ and $\tau_{\text {exc }}=\tau_{\text {rec }}=0.9 \mathrm{~ms}$. The of amplitudes were $v_{1}\left({ }^{1} \mathrm{H}\right)=100 \mathrm{kHz}$ and $v_{1}\left({ }^{14} \mathrm{~N}\right)=57 \mathrm{kHz}$. The projections onto the vertical $\omega_{1}$ axes (thin curves) may be compared with simulations (bold curves on right-hand side) obtained for a hypothetical uniform excitation of 4180 crystallite orientations and rotor synchronization with quadrupolar parameters $C_{Q}=1.18 \mathrm{MHz}$ and $\eta_{Q}=0.5$. The two-dimensional spectra result from averaging 128 (SQ) and 256 (DQ) transients for each of $256 \mathrm{t}_{1}$ increments with $\Delta \mathrm{t}_{1}=1 / \mathrm{v}_{\text {rot }}=33.3 \mu \mathrm{s}$, and a relaxation interval of $2 \mathrm{~s}$. (Reprinted with permission from ref. [33]. Copyright 2007 Elsevier Inc.)

incomplete averaging may occur, leading to a residual first-order line broadening. As SQ coherences are affected by first-order quadrupolar interactions while DQ coherences are not, ${ }^{[34]}$ a comparison between SQ and DQ spectra may reveal the presence of dynamics. We have used this approach to study local dynamics in polycrystalline samples of the tripeptide Ala-Ala-Gly (AAG). ${ }^{[24]}$ Nitrogen-14 SQ and DQ spectra (Fig. 3) were obtained at natural isotopic abundance via the proton-detected HMQC method presented in the previous section. The ${ }^{14} \mathrm{~N}$ quadrupolar couplings $C_{O}$ for the $\mathrm{NH}_{3}{ }^{+}$ammonium group (peak I in Fig. 3) and the two degenerate amide $\mathrm{NH}$ pairs (peak II in Fig. 3) were estimated to be +1.13 and $-3.21 \mathrm{MHz}$, respectively, by comparing the projection of the DQ spectrum onto the $\omega_{1}$ dimension with numerical simulations. ${ }^{[31]}$ On the other hand, the ${ }^{14} \mathrm{~N}$ SQ powder pattern of the $\mathrm{NH}_{3}^{+}$ammonium group exhibits significant broadening in comparison to the simulations obtained without including dynamics. In AAG, this broadening may be ascribed to oscillations of the N-terminal $\mathrm{N}-\mathrm{C}$ vector through small angles $\left(\sim 10^{\circ}\right)$ which would change the orientation of the ${ }^{14} \mathrm{~N}$ quadrupole tensor. Such dynamics would lead to jumps in the SQ precession frequencies that may interfere with the averaging of the first-order quadrupolar interaction under MAS. To test this hypothesis, we have run numerical simulations following the theory of McConnell[ ${ }^{[35-38]}$ for a symmetrical chemical exchange process involving two equally-populated states with a first-order exchange rate $k$. We have assumed $\pm 5^{\circ}$ jumps about the $y$ axis of the quadrupole tensor. In order to take into account the effects of the dynamics the simulations were carried 


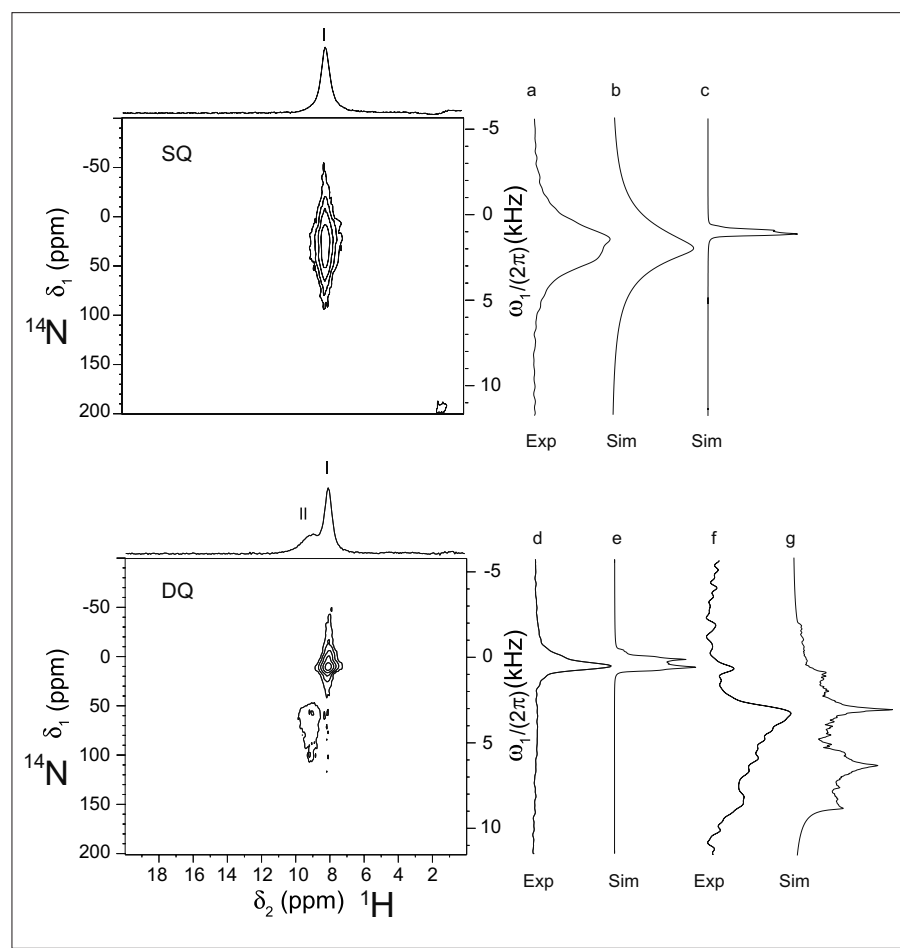

Fig. 3. Experimental $S Q$ (top) and $D Q$ (bottom) ${ }^{14} \mathrm{~N} /{ }^{1} \mathrm{H} H M Q C$ spectra of the $\mathrm{NH}_{3}{ }^{+}$group (I) and the two relatively rigid amide $\mathrm{NH}$ pairs that contribute to peak (II) in the tripeptide Ala-Ala-Gly (AAG). A $11 \mu$ sample in a $2.5 \mathrm{~mm}$ rotor was spun at $29.76 \mathrm{kHz}$ in a static field of $18.8 \mathrm{~T}(57.8$ and $800 \mathrm{MHz}$ for ${ }^{14} \mathrm{~N}$ and ${ }^{1} \mathrm{H}$, respectively). The if amplitudes were $\left.\mathrm{v}_{1}{ }^{1} \mathrm{H}\right)$ $=100 \mathrm{kHz}$ and $v_{1}\left({ }^{14} \mathrm{~N}\right)=80 \mathrm{kHz}$, except during the symmetry-based RN sequences used for recoupling in the excitation and refocusing intervals $\tau_{\text {exc }}=\tau_{\text {rec }}=100.8 \mu$ s when the proton $r f$ amplitude was set to $v_{1}\left({ }^{1} H\right)_{R N}=$ $59.52 \mathrm{kHz}$. More details about the recoupling method can be found in ref. [31]. The optimum ${ }^{14} \mathrm{~N}$ pulse lengths were $\tau_{\mathrm{p}}=20$ and $26 \mu$ s respectively for the $S Q$ and $D Q$ spectra. a) Experimental cross-section parallel to the $\omega_{1}$ dimension of the $S Q$ spectrum of site (I). b) Simulated $S Q$ response for a $\pm 5^{\circ}$ two-site jump, calculated as explained in the text. c) Simulated $S Q$ spectrum obtained without internal motions. (d, f) Experimental crosssections parallel to the $\omega_{1}$ dimension of the DQ spectrum of sites (I) and (II). (e, g) Simulated DQ spectra of sites (I) and (II), without motions. All simulations included 28656 crystallite orientations. The two-dimensional spectra resulted from averaging 256 (SQ) and 512 (DQ) transients for each of $100 \mathrm{t}_{1}$ increments with $\Delta \mathrm{t}_{1}=1 / \mathrm{v}_{\text {rot }}=33.6 \mu \mathrm{s}$, and a relaxation interval of 10 s. (Reprinted with permission from ref. [24]. Copyright 2008 American Chemical Society.)

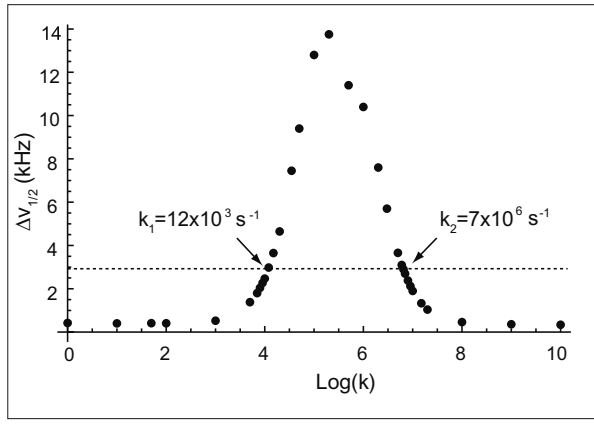

Fig. 4. Simulations of the full linewidth at halfheight $\Delta v_{1 / 2}$ of $S Q{ }^{14} \mathrm{~N}$ spectra as a function of the exchange rate $k$, assuming a two-site jump about the $y$ axis of the quadrupole tensor through $\pm 5^{\circ}, C_{O}=1.13 \mathrm{MHz}, \eta_{\odot}=0.28$, and 28656 different crystallite orientations. Only $0.4 \mathrm{kHz}$ of the width is due to the secondorder quadrupole splitting as shown in the simulation of Fig. 3c. The dashed horizontal line represents the width $\Delta v_{1 / 2} \approx 3 \mathrm{kHz}$ of the experimental SQ spectrum of Fig. 3a, after subtracting $0.2 \mathrm{kHz}$ due to proton-proton dipolar interactions determined from proton echo experiments which gave $\mathrm{T}_{2}{ }^{\prime}\left({ }^{1} \mathrm{H}\right)=1.6$ ms. (Reprinted with permission from ref. [24]. Copyright 2008 American Chemical Society.)

out in a Liouville space. In Fig. 4 we plot the simulated full linewidths at half-height $\left(\Delta v_{1 / 2}\right)$ as a function of the exchange rate $k$. One can see that the numerical simulations match the experimental SQ linewidth $\left(\Delta v_{1 / 2} \exp _{3 \mathrm{kHz}}\right)$ for either $k \approx 10^{4}$ or $10^{7}$ $\mathrm{s}^{-1}$. Experiments performed at different temperatures allow us to discriminate between these two possible regimes. We observed that the linewidth decreases with increasing temperature, thus excluding the slow regime. This proves unambiguously the presence of dynamic processes occur- ring at a rate $k \approx 10^{7} \mathrm{~s}^{-1}$. It is important to remark that this regime was previously not accessible to any spectroscopic method that provides atomic resolution.

\section{Acknowledgements}

The author is indebted to Prof. G. Bodenhausen, Dr. S. Cavadini and Dr. Anuji Abraham. This work was supported by the Fonds National de la Recherche Scientifique (FNRS) and the Commission pour la Technologie et l'Innovation (CTI), Switzerland.

Received: January 18, 2010

[1] F. Castellani, B. van Rossum, A. Diehl, M. Schubert, K. Rehbein, H. Oschkinat, Nature 2002, $420,98$.

[2] C. P. Jaroniec, C. E. MacPhee, V. S. Bajaj, M. T. McMahon, C. M. Dobson, R. G. Griffin, Proc. Nat. Acad. Sci. USA 2004, 101, 711.

[3] R. Tycko, Annu. Rev. Phys. Chem. 2001, 52, 575 .

[4] M. Ernst, A. Samoson, B. H. Meier, Chem. Phys. Lett. 2001, 348, 293.

[5] M. Ernst, A. Samoson, B. H. Meier, J. Magn Reson. 2003, 163, 332.

[6] M. Weingarth, P. Tekely, G. Bodenhausen, Chem. Phys. Lett. 2008, 466, 247.

[7] R. Blinc, M. Mali, R. Osredkar, A. Prelesnik, J. Seliger, I. Zupancic, L. Ehrenberg, J. Chem. Phys. 1972, 57, 5087.

[8] D. T. Edmonds, C. P. Summers, J. Magn. Reson. 1973, 12,134

[9] S. Cavadini, Prog. Nucl. Magn. Reson. Spectrosc. 2010, 56,46

[10] T. Giavani, H. Bildsoe, J. Skibsted, H. J. Jakobsen, J. Phys. Chem. B 2002, 106, 3026.

[11] S. Cavadini, S. Antonijevic, A. Lupulescu, G. Bodenhausen, ChemPhysChem 2007, 8, 1363.

[12] S. Cavadini, A. Lupulescu, S. Antonijevic, G. Bodenhausen, J. Am. Chem. Soc. 2006, 128, 7706

[13] Z. Gan, J. Am. Chem. Soc. 2006, 128, 6040.

[14] S. Cavadini, S. Antonijevic, A. Lupulescu, G. Bodenhausen, J. Magn. Reson. 2006, 182, 168.

[15] L. Müller, J. Am. Chem. Soc. 1979, 101, 4481.
[16] G. Bodenhausen, D. J. Ruben, Chem. Phys. Lett. 1980, 69, 185 .

[17] A. G. Palmer, J. Williams, A. McDermott, $J$. Phys. Chem. 1996, 100, 13293.

[18] J. R. Long, B. Q. Sun, A. Bowen, R. G. Griffin, J. Am. Chem. Soc. 1994, 116, 11950.

[19] S. E. Ashbrook, S. Antonijevic, A. J. Berry, S. Wimperis, Chem. Phys. Lett. 2002, 364, 634.

[20] S. P. Brown, M. Perez-Torralba, D. Sanz, R. M. Claramunt, L. Emsley, J. Am. Chem. Soc. 2002, $124,1152$.

[21] N. Giraud, M. Blackledge, M. Goldman, A. Böckmann, A. Lesage, F. Penin, L. Emsley, J. Am. Chem. Soc. 2005, 127, 18190.

[22] M. Cutajar, S. E. Ashbrook, S. Wimperis, Chem. Phys. Lett. 2006, 423, 276.

[23] M. J. Thrippleton, M. Cutajar, S. Wimperis, Chem. Phys. Lett. 2008, 452, 233.

[24] S. Cavadini, A. Abraham, S. Ulzega, G Bodenhausen, J. Am. Chem. Soc. 2008, 130 , 10850.

[25] T. G. Oas, R. G. Griffin, M. H. Levitt, J. Chem Phys. 1988, 89, 692.

[26] Z. H. Gan, D. M. Grant, Chem. Phys. Lett. 1990, 168, 304

[27] Z. H. Gan, D. M. Grant, R. R. Ernst, Chem. Phys. Lett. 1996, 254, 349

[28] Z. Gan, J. P. Amoureux, J. Trébosc, Chem. Phys. Lett. 2007, 435, 163.

[29] M. Carravetta, M. Eden, X. Zhao, A. Brinkmann, M. H. Levitt, Chem. Phys. Lett. 2000, 321, 205.

[30] A. Brinkmann, M. H. Levitt, J. Chem. Phys. 2001, 115, 357

[31] S. Cavadini, A. Abraham, G. Bodenhausen, Chem. Phys. Lett. 2007, 445, 1.

[32] S. Cavadini, V. Vitzthum, S. Ulzega, A. Abraham, G. Bodenhausen, J. Magn. Reson. 2010, 202, 57.

[33] S. Cavadini, A. Abraham, G. Bodenhausen, J. Magn. Reson. 2008, 190, 160

[34] A. Pines, D. J. Ruben, S. Vega, M. Mehring, Phys. Rev. Lett. 1976, 36, 110.

[35] H. M. McConnell, J. Chem. Phys. 1958, 28, 430.

[36] M. Helgstrand, T. Hard, P. Allard, J. Biomol. NMR 2000, 18, 49

[37] F. H. Larsen, J. Magn. Reson. 2004, 171, 293.

[38] F. H. Larsen, Solid State Nucl. Magn. Reson. 2007, 31, 100 . 